\title{
Aprimoramento do método h-M para estratificação vertical de uma floresta com araucária
}

\section{h-M method improvement for vertical stratification of an Araucaria forest}

\author{
Anke Manuela Salzmann' \\ Ana Paula Dalla Corte ${ }^{2}$ \\ Carlos Roberto Sanquetta ${ }^{3}(*)$
}

Resumo

Este trabalho teve como objetivo avaliar a estratificação vertical de um remanescente de Floresta Ombrófila Mista na região sul do Paraná. O estudo foi realizado com base em dados provenientes de oito parcelas amostrais permanentes, instaladas nos municípios de General Carneiro, Coronel Domingos Soares e Bituruna, para duas formações distintas sendo: campo com sub-bosque pouco desenvolvido e ralo e floresta densa. Para que o estudo da estratificação se tornasse possível, foram estimadas alturas para as araucárias e para o grupo das folhosas. A estimativa deu-se com base no ajuste de equações para as araucárias e folhosas independentemente. Para a representação da estratificação vertical da floresta, utilizou-se o método h-M, levando em consideração dados de diâmetro e altura coletados em 1998 e 2003. Em trabalhos anteriores o mesmo apresentou dificuldades na visualização da estratificação, não gerando picos abruptos entre um estrato e outro. Foi elaborada, portanto, uma metodologia que proporcionasse uma divisão mais abrupta entre os estratos, através da divisão do banco de dados em três grupos. A metodologia proposta indicou para a formação de campo com sub-bosques pouco desenvolvidos, a existência de três estratos em 1998 e quatro em 2003. Para a floresta densa foram observados para ambos os anos cinco estratos verticais.

Palavras-chave: estratos florestais; equações hipsométricas; Floresta Ombrófila Mista; Paraná.

I Graduanda em Engenharia Florestal na Universidade Federal do Paraná, UFPR; Curitiba, Paraná, Brasil; Bolsista do Programa de Ensino Tutorial, PET; E-mail: ankemanu@yahoo.com.br

2 Dra.; Engenheira Florestal; Pesquisadora em projetos de pesquisas desenvolvidos pelas Fundações conveniadas com a Universidade Federal do Paraná, UFPR; Curitiba, Paraná, Brasil; E-mail: anapaulacorte@ gmail.com

$3 \mathrm{PhD}$; Engenheiro Florestal; Professor/Pesquisador da Fundação de Pesquisas Florestais do Paraná, FUPEF e do Departamento de Ciências Florestais da Universidade Federal do Paraná, UFPR; Bolsista de Produtividade em Pesquisa do CNPq; Endereço: Avenida Lothário Meissner, 900, Jardim Botânico, CEP: 80210-170, Curitiba, Paraná, Brasil; E-mail: sanquetta@ufpr.br (*) Autor para correspondência.

Recebido para publicação em 22/07/2010 e aceito em I8/10/201।

\begin{tabular}{llllll}
\hline Ambiência Guarapuava (PR) & v.7 n.3 & p. 575-586 Set./Dez. 20II & ISSN I808 - 025 I
\end{tabular}

DOI: 10.5777/ambiencia. 2011.03.12 


\section{Abstract}

The purpose of this paper was to assess the vertical stratification of an Araucaria Forest in the south of Paraná. The study was based on data from eight permanent sample plots, established in the counties of General Carneiro, Coronel Domingos Soares and Bituruna, which are comprised of two different forest types: woodland (open canopy with grassland in the understory) and closed canopy. In order to enable the stratification, heights were estimated for the araucaria trees and broad-leaved species. The tree height estimation was based on the adjustment of independent equations for araucaria and broad-leaved trees. For the representation of the vertical forest stratification the h-M method was applied, taking into consideration DBH and height data, collected in 1998 and 2003. Previous studies, which used this method, have shown some practical difficulties regarding vertical stratification definition, considering that the peaks between a stratum and another could not be easily identified. Therefore, an improvement of the $\mathrm{h}-\mathrm{M}$ method was proposed in this research, which allowed a more visible and abrupt separation between strata, through the division of the data bank into three groups. The proposed methodology indicated three and four strata for the woodland (open canopy with grassland in the understory) in 1998 and 2003, respectively. For the closed canopy forest type five vertical stories were identified in both years.

Key words: forest strata; dbh-height equations; Araucaria Forest; Paraná.

\section{Introdução}

A Floresta Ombrófila Mista, também conhecida como "Pinheirais" ou Floresta com Araucária, é um subgrupo da Floresta Atlântica que está circunscrito a uma região de clima pluvial subtropical, ocorrendo em altitudes superiores a 800 metros s.n.m., predominantemente no sul do Brasil. No Paraná, a região fitoecológica da Floresta Ombrófila Mista principia no Primeiro Planalto, a oeste da Serra do Mar, estendendo-se pelos Segundo e Terceiros Planaltos (PRÓ-ATLÂNTICA, 2005).

Esta exuberante tipologia considerada um dos mais importantes ecossistemas brasileiros, cobria originalmente uma área superior a $182.000 \mathrm{~km}^{2}$ (SANQUETTA e TETTO, 2000), mas atualmente seus remanescentes primários são escassos, restando florestas secundárias alteradas pela intensa atividade antrópica. Brepohl (1980) relata que as áreas de Floresta de Araucária abrangiam inicialmente cerca de $73.780 \mathrm{~km}^{2}$ do Estado do Paraná. O início da exploração do pinheiro (Araucaria angustifolia Bert. O. Ktze), espécie característica da Floresta Ombrófila Mista, remonta ao século XVIII, quando a Coroa Portuguesa descobriu o potencial que ofereciam a madeira de boa qualidade e o tronco reto da árvore (SANQUETTA; TETTO, 2000).

Desde então, esta floresta, sofreu um processo de exploração intensiva. As densas florestas tropicais eram tidas aos olhos dos colonizadores e, mais tarde, dos proprietários de terras, como inesgotáveis. Esse processo fez-se sentir de maneira particularmente 
grave no caso das áreas de domínio da Floresta Atlântica, principalmente no Sudeste e Sul do país (ENGEL, 1999).

Hoje os remanescentes em estágio avançado de sucessão, somam, segundo (SANQUETTA, 2005), menos de 1\% da área que existia originalmente no Paraná. A proteção desses fragmentos tornou-se um grande desafio para a sociedade, requerendo medidas legislativas e de fiscalização rígidas. Porém, ações de caráter científico, que possibilitem o conhecimento do ecossistema tornando a recomendação e execução de práticas de conservação e manejo possíveis, são ainda mais imprescindíveis.

Ainda restam 2,7 milhões de hectares de florestas em estágios inicial e médio de sucessão que estão nas mãos de proprietários rurais que precisam ser instruídos de forma a tratar adequadamente as suas áreas (SANQUETTA, 2005). O manejo dessas florestas, para fins produtivos e conservacionistas, pode ser uma alternativa viável (SANQUETTA; MATTEI, 2002). Entretanto, para manejar racionalmente as florestas alteradas por ações antrópicas, é preciso conhecer o seu processo dinâmico e acima de tudo estrutural. A floresta é composta por uma estrutura vertical, componente muito importante que cumpre um papel fundamental nos processos ecológicos, na produção de biomassa e na coexistência de espécies (KOHYAMA, 1991). A avaliação da estrutura vertical é importante, uma vez que pode ser de grande valia para o manejo (SANQUETTA, 1995).

Ainda são poucos os estudos desenvolvidos sobre a estruturação vertical de uma Floresta Ombrófila Mista. Sanquetta et al. (2002) estudaram a estrutura vertical de um fragmento de Floresta com Araucária no centro-sul do Paraná, através do método
h-M. Este foi desenvolvido por Sanquetta em 1995 para possibilitar a análise estrutural de florestas. O presente método permite reconhecer os diferentes estratos presentes em povoamentos e em populações específicas, onde a variável altura é inserida como indicadora da estratificação. Os gráficos gerados indicam através de picos, a divisão entre os estratos existentes na floresta estudada. Em alguns casos, os picos não são muito claros, tornando o reconhecimento dos diferentes estratos complicada e imprecisa.

O objetivo deste trabalho foi estudar e apresentar uma metodologia que aprimorasse o método $\mathrm{h}-\mathrm{M}$, proporcionando uma visualização mais clara e definida da estratificação florestal gerada pelos diagramas h-M. Com base na metodologia proposta, foi analisada a estrutura vertical de um remanescente de Floresta Ombrófila Mista na região sul do Paraná, quantificando o número de estratos existentes em uma tipologia de campo com sub-bosque ralo e pouco desenvolvido e em outra de floresta densa.

\section{Material e métodos}

O estudo foi realizado em remanescentes de Floresta com Araucária, na propriedade das Indústrias Pedro N. Pizzatto Ltda., localizada no município de General Carneiro, no extremo sul do Paraná, próximo à divisa com o estado de Santa Catarina, a cerca de $280 \mathrm{~km}$ de Curitiba A altitude regional está em torno de $800 \mathrm{~m}$ e as coordenadas geográficas de referência são $26^{\circ} 20^{\prime} 35^{\prime \prime}$ e $26^{\circ} 26^{\prime} 13^{\prime \prime}$ Latitude Sul, e 51'19'49” e 5125'29” Longitude Oeste. Segundo a EMBRAPA (1999), a área em estudo é composta de três tipos de solos: CAMBISSOLOS, NEOSSOLOS e LITOSSOLOS. 
O clima, segundo a classificação de KÖPPEN, é do tipo Cfb, que é característico de regiões frias e típico dos planaltos paranaenses de clima subtropical úmido mesotérmico (MAACK, 1968), cuja temperatura média é inferior a $22^{\circ} \mathrm{C}$ durante o mês mais quente, sem estação seca, com verões frescos e mais de cinco geadas noturnas por ano (PARANÁ, 1987).

Os remanescentes estudados pertencem à tipologia de Floresta Ombrófila Mista, conforme classificação do IBGE (1992), onde predominam como espécies arbóreas o pinheiro-do-paraná (Araucaria angustifolia), a erva-mate (Ilexparaguariensis), a imbuia (Ocotea porosa), a bracatinga (Mimosa scabrella), as canelas (Ocotea spp. e Nectandra spp. principalmente), diversas espécies da família Myrtaceae, dentre outras.

Para o aprimoramento do método h-M, foi estudado um banco de dados referente às medições de $\mathrm{CAP}$ (circunferência a altura do peito) de árvores pertencentes a oito parcelas amostrais permanentes do Programa Ecológico de Longa Duração (PELD) que vêem sendo remedidas há oito anos. Cada parcela mede 1,00 ha $(100 \times 100$ $\mathrm{m})$, sendo subdividida em 25 subparcelas de $400 \mathrm{~m}^{2}$ (20 m x $20 \mathrm{~m}$ ). As parcelas 1 e 4 encontram-se em uma área de campo com um sub-bosque pouco desenvolvido e ralo. O restante das parcelas $(2,3,5,6$, 7 e 8) apresenta características de floresta densa, sendo a estratificação analisada para estas duas formações. As medições ocorrem anualmente na área, sendo medidos todos os indivíduos que apresentarem o diâmetro mínimo de inventário estipulado em 10 $\mathrm{cm}$, o que equivale a um CAP de $31,4 \mathrm{~cm}$. Todas as árvores medidas foram devidamente identificadas em nível de gênero e espécie. A análise da estratificação vertical da floresta em questão, deu-se para um período de cinco anos, ou seja, de 1998, início das medições, até 2003.

Foram coletados valores de altura real das árvores para o ajuste de equações hipsométricas, a fim de possibilitar a estimativa da altura para os indivíduos que compõem o conjunto de dados citado acima. Foram ajustadas duas equações hipsométricas, uma para as araucárias, por apresentarem um formato característico e uma boa correlação entre diâmetro e altura, e outra, para as demais árvores classificadas como folhosas. A obtenção das alturas reais deu-se através da utilização dos hipsômetro de Haga.

O ajuste da equação para as araucárias baseou-se nas medidas de altura real obtidas para 78 indivíduos, enquanto que para as folhosas o número de indivíduos amostrados foi de 170 . No caso das folhosas a amostragem foi maior, por se tratar de um grupo que englobava mais de uma espécie.

Para o ajuste das equações referentes às araucárias foram testados os modelos hipsométricos representados na tabela 1 , que também foram testados por Pizatto (1999) em seu trabalho sobre avaliação biométrica da estrutura e dinâmica de uma Floresta Ombrófila Mista no Paraná. Para as folhosas, foram testados os modelos apresentados na Tabela 1 , sendo os quatro primeiros também testados por Pizatto (1999). A escolha do melhor modelo baseou-se nos valores do coeficiente de determinação $\left(\mathrm{R}^{2}{ }_{\text {ajust. e recalculado }}\right)$, erro padrão da estimativa $\left(\mathrm{S}_{\mathrm{yx}} \%_{\text {recalculado }}\right)$, bem como no gráfico de distribuição dos resíduos. Após escolhida a equação mais adequada para cada caso, foi estimada a altura para os demais indivíduos pertencentes às oito parcelas permanentes. 
Tabela I. Modelos hipsométricos testados para as araucárias e para as folhosas

\begin{tabular}{cc}
\hline Modelos testados para Araucária & Modelos testados para Folhosas \\
\hline $1 . \ln h=\mathrm{b}_{0}+\mathrm{b}_{1}(d)$ & $1 . \ln h=\mathrm{b}_{0}+\mathrm{b}_{1}(\mathrm{~d})$ \\
$2 . \ln h=\mathrm{b}_{0}+\mathrm{b}_{1}(1 / d)$ & $2 . \ln h=\mathrm{b}_{0}+\mathrm{b}_{1}(1 / d)$ \\
$3.1 /(h-1.30)=\mathrm{b}_{0}+\mathrm{b}_{1}(1 / \mathrm{d})$ & $3.1 /(h-1.30)=\mathrm{b}_{0}+\mathrm{b}_{1}(1 / \mathrm{d})$ \\
$4 . h-1.30=d^{2} /\left(\mathrm{b}_{0}+\mathrm{b}_{1}(d)+\mathrm{b}_{2}\left(d^{2}\right)\right)$ & $4 . h-1.30=d^{2} /\left(\mathrm{b}_{0}+\mathrm{b}_{1}(d)+\mathrm{b}_{2}\left(d^{2}\right)\right)$ \\
- & $5 \cdot h=\mathrm{b}_{1} \ln (d)-\mathrm{b}_{0}$ \\
\hline
\end{tabular}

Após a estimativa das alturas, foram gerados os diagramas $h-M$. Neste caso " $h$ " refere-se à altura total estimada das árvores e "M" representa a média cumulativa parcial da altura a partir do maior valor de h, dada pelo quociente da soma das alturas das árvores a partir do maior valor de $\mathrm{h}$ e da soma do número de árvores até um valor de $\mathrm{h}$ inferior ao valor máximo de $\mathrm{h}$. Os gráficos obtidos foram interpretados e em seguida, foi elaborada uma metodologia que permitisse uma visualização mais definida da estratificação florestal gerada pelos diagramas h-M, através da formação abrupta de picos entre um estrato e outro.

Para que a diferenciação entre os estratos se tornasse mais visível, o conjunto de árvores estudado foi dividido em três grupos contendo o mesmo número de indivíduos, sendo:

Grupo I: árvores mais baixas;

Grupo II: árvores de altura média;

Grupo III: árvores mais altas.

Após a definição dos grupos, o conjunto de dados foi manipulado a fim de proporcionar um pico mais abrupto entre um estrato e outro. Do grupo I foi retirado um número de árvores equivalente a 10\% das árvores mais baixas, no grupo II foram retirados 5\% das árvores mais altas e 5\% dos indivíduos mais baixos dentro deste mesmo grupo e no grupo III, foi retirado o equivalente a $10 \%$ das árvores mais altas. Após a manipulação do banco de dados, foram gerados novos diagramas $\mathrm{h}-\mathrm{M}$ onde se pôde avaliar o desempenho deste método modificado.

\section{Resultados e Discussões}

\section{Equações ajustadas}

A análise da tabela 2 , bem como da figura 1, permitiu concluir que a melhor equação para a estimativa da altura total para as araucárias neste caso, foi a de número 4 , $h-1,30=d^{2} /(-9,42323+1,83039(d)+0,02440$ $\left.\left(d^{2}\right)\right)$. Esta apresentou os melhores resultados de ajuste, precisão e distribuição de resíduos, resultando em um $\mathrm{R}_{\text {ajustado recalculado }}$ de 0,50835 e Syx recalculado $_{0} \%$ de $14,52 \%$.

De forma geral, as menores classes de diâmetro apresentaram maior variação dos resíduos, porém, não foi identificada tendência que impedisse a aplicação do modelo selecionado. Foram observados alguns poucos outliers na distribuição gráfica dos resíduos. 
Tabela 2. Resultados dos modelos hipsométricos testados para as araucárias

\begin{tabular}{ccccc}
\hline Modelo & Coeficientes & $\mathrm{R}_{\text {ajustado recalculado }}^{2}$ & Syx $_{\text {recalculado }}$ & Syx $_{\text {recalculado }}$ \% \\
\hline 1) $\ln b=\mathrm{b}_{0}+\mathrm{b}_{1}(d)$ & $\begin{array}{r}\mathrm{b}_{0}=1,25882 \\
\mathrm{~b}_{1}=0,40935\end{array}$ & 0,34503 & 3,21 & 18,67 \\
\hline 2) $\ln h=\mathrm{b}_{0}+\mathrm{b}_{1}(1 / d)$ & $\begin{array}{l}\mathrm{b}_{0}=2,98519 \\
\mathrm{~b}_{1}=-7,97411\end{array}$ & 0,22102 & 3,55 & 20,67 \\
\hline 3) $1 /(h-1.30)=\mathrm{b}_{0}+\mathrm{b}_{1}(1 / \mathrm{d})$ & $\begin{array}{l}\mathrm{b}_{0}=0,04790 \\
\mathrm{~b}_{1}=0,65852\end{array}$ & 0,37277 & 3,13 & 18,19 \\
\hline 4) $h-1.30=d^{2} /\left(\mathrm{b}_{0}+\mathrm{b}_{1}(d)+\mathrm{b}_{2}\left(d^{2}\right)\right)$ & $\begin{array}{l}\mathrm{b}_{0}=-9,42323 \\
\mathrm{~b}_{1}=1,83039\end{array}$ & & & \\
\hline $\mathrm{b}_{2}=0,02440$ & & & \\
\hline
\end{tabular}

\section{Onde:}

$h=$ altura total estimada (metros);

$d$ = Diâmetro à altura do peito (centímetros).

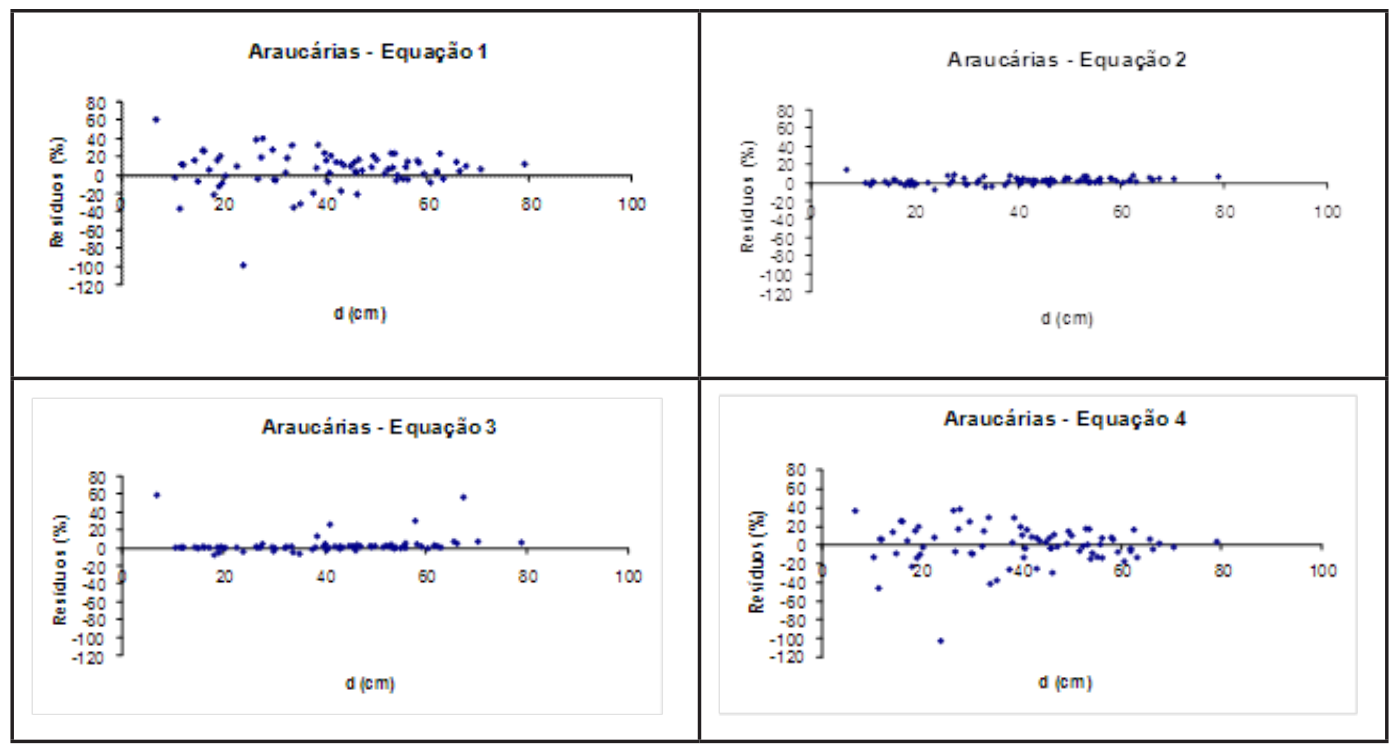

Figura I. Distribuição de resíduos dos modelos ajustados para estimativa de altura total para as araucárias

A análise da tabela 3, bem como da figura 2 permitiu concluir que a melhor equação para a estimativa da altura total para as folhosas neste trabalho, foi a equação logarítmica de número 5 , $h=6,18290 \ln (d)-(-7,26910)$. Esta apresentou os melhores resultados de ajuste, precisão e distribuição de resíduos, resultando em um $\mathrm{R}^{2}{ }_{\text {ajustado recalculado }}$ de 0.3894 e Syx ${ }_{\text {recalculado }} \%$ de $31,08 \%$. De forma geral, as menores classes de diâmetro apresentaram maior variação dos resíduos, porém, não foi identificada tendência que impedisse a aplicação do modelo selecionado. 
Tabela 3. Resultado para os modelos hipsométricos testados para as folhosas

\begin{tabular}{ccccc}
\hline Modelo & Coeficientes & $\mathbf{R}_{\text {ajustado recalculado }}^{\mathbf{2}}$ & $\mathbf{S y x}_{\text {recalculado }}$ & Syx $_{\text {recalculado }}$ \% \\
\hline 1) $\ln h=\mathrm{b}_{0}+\mathrm{b}_{1}(\mathrm{~d})$ & $\begin{array}{l}\mathrm{b}_{0}=-0,10815 \\
\mathrm{~b}_{1}=0,76284\end{array}$ & 0,10235 & 3,66 & 36,23 \\
\hline 2) $\ln h=\mathrm{b}_{0}+\mathrm{b}_{1}(1 / d)$ & $\begin{array}{c}\mathrm{b}_{0}=2,86529 \\
\mathrm{~b}_{1}=-12,73127\end{array}$ & 0,10235 & 3,66 & 36,23 \\
\hline 3) $1 /(b-1.30)=\mathrm{b}_{0}+\mathrm{b}_{1}(1 / \mathrm{d})$ & $\begin{array}{l}\mathrm{b}_{0}=-0,00860 \\
\mathrm{~b}_{1}=2,57883\end{array}$ & 0,03520 & 3,82 & 37,75 \\
\hline 4) $b-1.30=d^{2} /\left(\mathrm{b}_{0}+\mathrm{b}_{1}(d)+\mathrm{b}_{2}\left(d^{2}\right)\right)$ & $\begin{array}{l}\mathrm{b}_{0}=41,62501 \\
\mathrm{~b}_{2}=-2,39615\end{array}$ & 0,19136 & 3,30 & 32,64 \\
\hline 5$) h=\mathrm{b}_{1} \ln (d)-\mathrm{b}_{0}$ & $\begin{array}{l}\mathrm{b}_{0}=-7,26910 \\
\mathrm{~b}_{1}=6,18290\end{array}$ & 0,38940 & 3,12 & 31,08 \\
\hline
\end{tabular}

Onde:

$h=$ altura total estimada (metros);

$d=$ Diâmetro à altura do peito (centímetros).

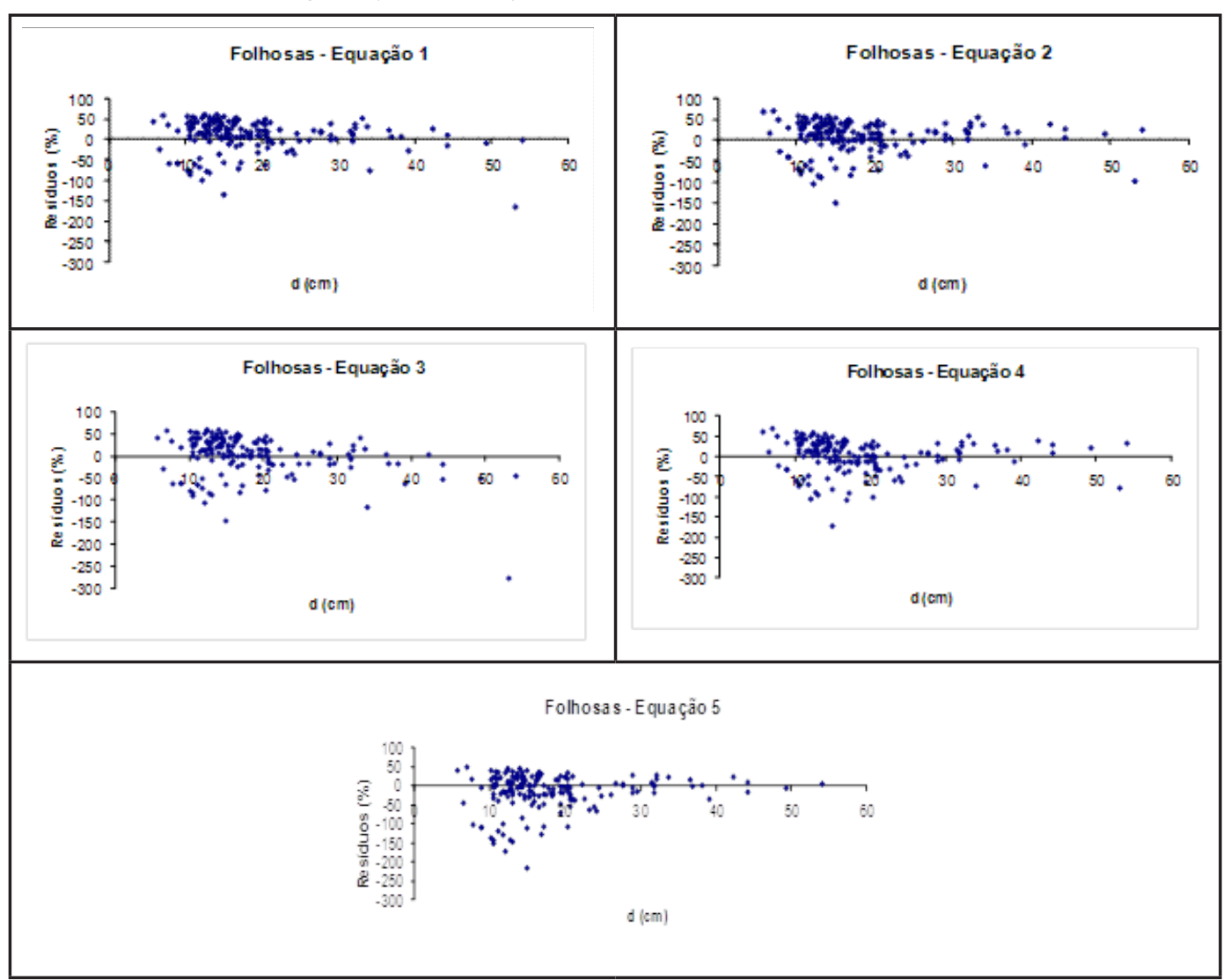

Figura 2. Distribuição de resíduos dos modelos ajustados para estimativa de altura total para as folhosas 
Os valores ajustados e recalculados foram menores para as folhosas por este grupo incluir uma quantidade muito grande de espécies com características ecológicas distintas. Para que o resultado de um ajuste de modelo hipsométrico para esta situação seja mais efetivo, seria necessário intensificar a amostragem e eventualmente trabalhar com grupos de espécies que possuam uma ecologia semelhante ou ainda, ajustar equações hipsométricas para cada espécie.

Outro problema que interferiu na obtenção de resultados mais precisos está relacionado a uma espécie comercial chamada erva-mate (Ilex paraguariensis). Esta árvore sofre podas anuais para a coleta de suas folhas que são comercializadas para a fabricação da erva utilizada no chimarrão. Estas podas influenciam no crescimento em altura da árvore e conseqüentemente na estimativa da altura total pela equação, visto que a mesma baseia-se no diâmetro para a estimativa da altura.

O ajuste da equação hipsométrica para a espécie araucária (Araucaria angustifolia), apresentou valores melhores por se tratar justamente de um grupo amostrado de uma única espécie. Neste caso não se corre o risco, como nas folhosas, de que determinadas espécies não sejam amostradas. Além disso, o processo de amostragem de indivíduos nas diversas classes de diâmetro e altura é facilitado, ao se trabalhar com apenas uma espécie.

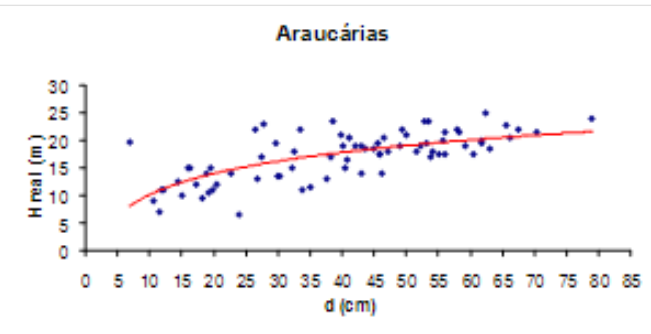

A correlação entre altura e diâmetro para as araucárias e folhosas, indicadas na figura 3 revela-se melhor distribuída para as araucárias, devido às questões discutidas acima. Para as folhosas, observa-se uma falta de amostragem para árvores de maiores diâmetros. Isso ocorre pelo fato de árvores de grandes diâmetros serem relativamente escassas, quando comparadas aos demais diâmetros, geralmente menores, encontrados na floresta estudada. A área já fora alvo de exploração de espécies madeireiras nobres no seu passado conforme reporta o trabalho de Pizatto (1990).

\section{Diagramas h-M}

Foram gerados diagramas h-M para a situação de campo com sub-bosque pouco desenvolvido e floresta densa, tanto para o ano de 1998, quanto para o ano de 2003, para fins comparativos (Figuras 4 a 7 ). Inicialmente os gráficos foram construídos com base nos dados brutos e em seguida, eles foram gerados a partir do banco de dados manipulado pela metodologia proposta para aprimoramento do método h-M.

$\mathrm{Na}$ figura 4 a estratificação existe no diagrma h-M 1998 - Dados Originais, entretanto, não está bem definida por picos abruptos entre um estrato e outro. Através da manipulação do banco de dados estudado, por meio da metodologia proposta, a distinção entre os estratos que compõem a tipologia

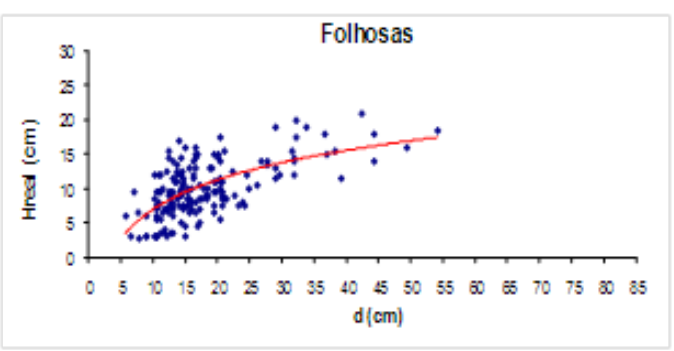

Figura 3. Correlação entre diâmetro e altura para as equações selecionadas 


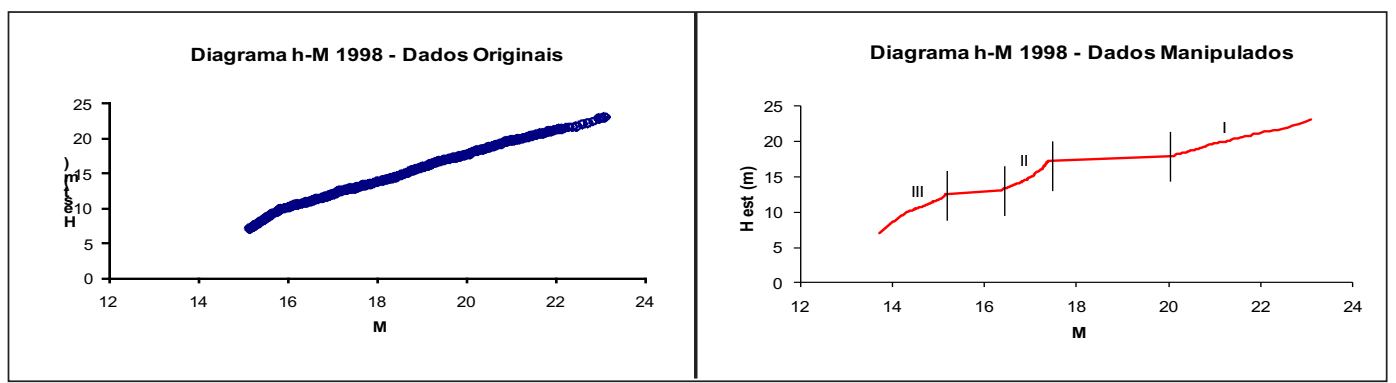

Figura 4. Diagrama h-M com dados originais e manipulados para a tipologia campo com sub-bosque ralo representada pelas parcelas I e 4 em 1998

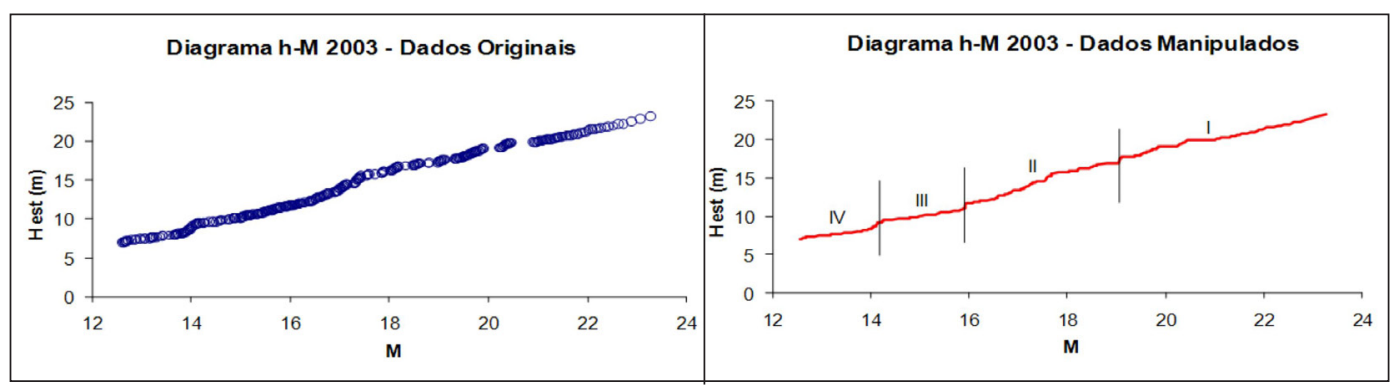

Figura 5. Diagrama h-M com dados originais e manipulados para a tipologia campo com sub-bosque ralo representada pelas parcelas I e 4 em 2003

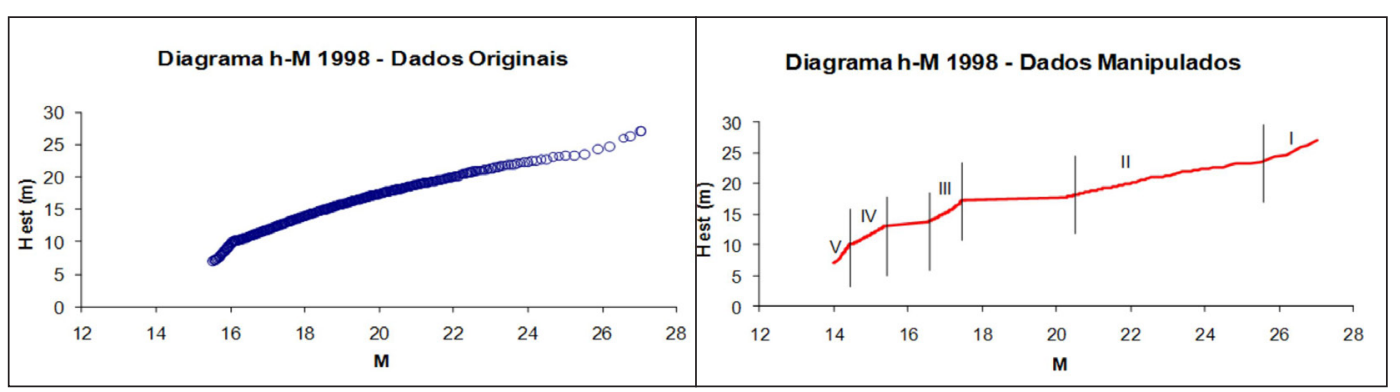

Figura 6. Diagrama h-M com dados originais e manipulados para a tipologia floresta densa representada pelas parcelas 2, 3, 5, 6, 7 e 8 em 1998

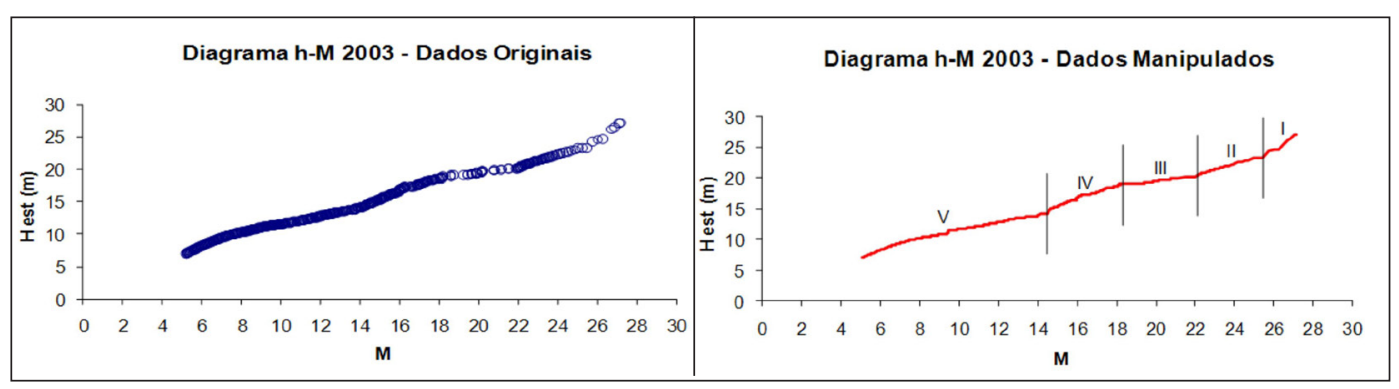

Figura 7. Diagrama h-M com dados originais e manipulados para a tipologia floresta densa representada pelas parcelas 2, 3, 5, 6, 7 e 8 em 2003 
campo, tornou-se mais clara. O diagrama h-M 1998 - Dados Manipulados indica que no mesmo ano, as parcelas inseridas em um ambiente de campo com sub-bosque ralo, apresentaram três estratos bem definidos. $\mathrm{O}$ primeiro estrato compreendeu árvores com alturas superiores a $17 \mathrm{~m}$, enquanto que o segundo contou com árvores entre 13 e 17 $\mathrm{m}$ e o terceiro e último, apresentou árvores inferiores a $12 \mathrm{~m}$.

$\mathrm{Na}$ figura 5 o diagrama h-M 2003 - Dados Originais revela picos um pouco mais visíveis, indicando a existência de três estratos. No diagrama h-M 2003 - Dados Manipulados observa-se a visualização de três picos para o ano de 2003, sendo possível observar um novo estrato para a formação de campo com sub-bosque ralo. Este diagrama apresenta, portanto, quatro estratos sendo: I - árvores superiores a $17 \mathrm{~m}$; II - árvores entre 11 e $17 \mathrm{~m}$; III - árvores entre 8 a 11 $\mathrm{m}$; IV - árvores inferiores a $8 \mathrm{~m}$. Mesmo com a manipulação do banco de dados, observa-se no diagrama h-M 2003 - Dados Manipulados, uma maior dificuldade de distinção entre estratos, principalmente na região que compreende os estratos I e II. Isso pode ser resultado da própria dinâmica florestal. Sanqueta et al. (2003) observaram em um estudo realizado na mesma área, durante o período de 1998 a 2002, altas taxas de recrutamento na floresta e consideráveis taxas de mortalidade para araucárias e imbuias adultas, espécies características dos estratos I e II. Isto indica que o ambiente estudado encontra-se em pleno processo de desenvolvimento, não estando a estratificação completamente definida ainda. $O$ surgimento de um novo estrato também representa o dinamismo e desenvolvimento contínuo da floresta, indicando o avanço da mesma sobre o campo.
Igualmente como nos dois casos anteriores, a visualização de estratos bem definidos no diagrama h-M 1998 - Dados Originais (Figura 6), é dificultada quando comparada ao diagrama h-M 1998 - Dados Manipulados presente na mesma figura. Este revelou a existência de cinco estratos na floresta estudada sendo a estratificação a seguinte: I - árvores superiores a $23 \mathrm{~m}$; II - árvores entre 18 e $23 \mathrm{~m}$; III - árvores de 14 a $17 \mathrm{~m}$; IV - árvores entre 9 e $13 \mathrm{~m} \mathrm{e}$ $\mathrm{V}$ - árvores inferiores a $9 \mathrm{~m}$. A diversidade maior em estratos, quando comparada às parcelas de campo com sub-bosque pouco desenvolvido, deve se ao próprio ambiente. A situação de floresta fechada apresenta uma densidade maior de indivíduos, assim como uma variedade maior de espécies cada qual com suas características ecológicas próprias, ocupando estratos distintos.

Assim como no diagrama h-M 1998 - Dados Originais representado na figura 6, o diagrama h-M 2003 - Dados Originais apresentado na figura 7 também revela uma divisão da área estudada em três estratos. Depois de manipulados os dados, é possível observar cinco estratos no diagrama h-M 2003 - Dados Manipulados, sendo dois destes, omitidos no diagrama criado a partir dos dados brutos. A estratificação indicada pelo diagrama h-M 2003 - Dados manipulados foi: I - árvores superiores a 23 m; II - árvores entre 20 a 23 m; III - árvores de 18 a 20 m; IV - árvores entre 14 e 18 m; $\mathrm{V}$-árvores inferiores a $14 \mathrm{~m}$. Comparandose os diagramas h-M - Dados Manipulados para a tipologia floresta densa em 1998 e 2003 (Figuras 6 e 7), observa-se que não surgiu um novo estrato na floresta estudada. Ocorreu uma mudança de altura dos estratos, com exceção do primeiro que manteve árvores com alturas superiores a $23 \mathrm{~m}$. 


\section{Conclusões}

A realização deste estudo permitiu concluir os seguintes pontos:

- O ajuste de equações hipsométricas por espécie alcançou resultados mais satisfatórios. Em ajuste de equações hipsométricas para um conjunto de diversas espécies há o risco da amostragem adotada, não contemplar adequadamente a realidade estudada;

- A metodologia proposta permitiu no diagrama h-M, a formação de picos mais abruptos entre um estrato e outro, facilitando a visualização da estratificação existente na floresta estudada;

- Para a formação de campo com subbosque pouco desenvolvido e ralo, observou-se em 1998 três estratos verticais; e em 2003 o surgimento de um quarto estrato, indicando o avanço da floresta sobre o campo limpo;

- Para a floresta densa, o número de estratos verticais observados tanto para 1998 como 2003, foi equivalente a cinco. Todos os estratos sofrerem mudanças em suas alturas, exceto o primeiro que manteve a altura máxima inalterada.

- Em caso de manejo da espécie ervamate (Ilex paraguariensis) para uso comercial, esta deverá ser desconsiderada no processo de coleta de dados para o ajuste de uma equação hipsométrica. A constante poda da mesma resulta em uma altura que não corresponde ao diâmetro da árvore, forçando uma superestimativa de altura neste caso.

\section{Referências}

BREPOHL, D. Análise da política de incentivos fiscais para o reflorestamento no Brasil e no Paraná. 1980. 216 f.Dissertação (Mestrado em Economia e Política Florestal) - Universidade Federal do Paraná, Curitiba, 1980.

ELGEL, V. L. Introdução aos Sistemas Agroflorestais. Botucatu: FEPAF, 1999. 70 p.

EMPRESA BRASILEIRA DE PESQUISA AGROPECUÁRIA - EMBRAPA. Centro Nacional de Pesquisa de Solos (Rio de Janeiro, RJ). Sistema Brasileiro de Classificação de Solos. Brasília: Embrapa Produção da Informação; Rio de Janeiro: Embrapa Solos, 1999. 412p.

INSTITUTO BRASILEIRO DE GEOGRAFIA E ESTATÍSTICA - IBGE. Manual técnico da vegetação brasileira. n.1. Rio de Janeiro: DEDIT/CDDI, 1992. 92p.

KOHYAMA, T. Simulating stationary size distribution of trees in rain forests. Annals of Botany, v.68, n.2, p. 173-180, 1991.

MAACK, R. Geografia física do Estado do Paraná. Curitiba: CODEPAR, 1968. 350p.

PARANÁ. Atlas do Estado do Paraná. Curitiba: Secretária de Estado de Agricultura e Abastecimento - Instituto de Terras, Cartografia e Florestas, 1987.73p.

PIZATTO, W. Avaliação biométrica da estrutura e da dinâmica de uma Floresta Ombrófila Mista em São João do Triunfo - PR: 1995 a 1998. 1999. 172 f. Dissertação (Mestrado em Ciências Florestais) - Universidade Federal do Paraná, UFPR, Curitiba, 1999. 
PRÓ-ATLÂNTICA. Atlas da Floresta Atlântica no Paraná. Curitiba: Pró-Atlântica/ SEMA Paraná, 2005. 104p.

SANQUETTA, C. R. Análise da estrutura vertical de florestas através do diagrama h-M. Ciência Florestal, Santa Maria, v. 5, n.1, p. 55-68, 1995.

SANQUETTA, C. R; TETTO, A. F. Pinheiro do Paraná: lendas e realidades. Curitiba: Fundação de Pesquisas Florestais do Paraná, 2000.112 p.

SANQUETTA, C. R.; MATTEI, E. Manejo racional da floresta de araucária. Florianópolis: Meio Ambiente. Santa Catarina, Florianópolis, v. 2, 2002.58 p.

SANQUETTA, C. R.; PIZATTO, W.; PÉLLICO NETTO, S.; FIGUEIREDO FILHO, A.; EISFELD, R. L. Estrutura vertical de um fragmento de Floresta Ombrófila Mista no centro-sul do Paraná. Floresta, Curitiba, v. 32, n. 2, p. 267-276, 2002.

SANQUETTA, C. R.; CÔRTE, A.P.D.; EISFELD R L. Crescimento, mortalidade e recrutamento em duas florestas de araucária (Araucaria angustifolia (Bert.) O. Ktze.) no estado do Paraná, Brasil. Revista Ciências Exatas e Naturais, Guarapuava, v.5, n.1, p.101-112, 2003.

SANQUETTA, C. R. Fragmentação da Floresta Ombrófila Mista no Paraná. In: SIMPÓSIO SOBRE A MATA ATLÂNTICA: Conservação, Recuperação e Desenvolvimento, 1., 2005, Viçosa. Anais... Viçosa: CBCN - Centro Brasileiro para Conservação da Natureza e Desenvolvimento Sustentável, 2005. 1 CD-ROM. 